Juraci A. Cesar ${ }^{1}$ Bernardo L. Horta ${ }^{2}$ Gildo Gomes ${ }^{1}$ Ricardo S. Houlthausen 3 Roselane M. Willrich 1 Alessandra Kaercher 1 Francisco M. Iastrenski 1

\section{Fatores associados à não realização de exame citopatológico de colo uterino no extremo Sul do Brasil}

\author{
Factors associated with non-participation in \\ screening for cervical cancer in Southern Brazil
}

\footnotetext{
1 Departamento Materno-Infantil, Universidade Federal do Rio Grande. Rua Engenheiro Alfredo Huch 475, Rio Grande, RS 96201-900, Brasil. jacesar@furg.br 2 Escola de Medicina, Universidade Católica de Pelotas. Rua Félix da Cunha 412, Pelotas, RS 96010-000, Brasil. 3 Faculdade de Medicina, Universidade Federal de Pelotas. Av. Duque de Caxias 250, Pelotas, RS 96001-970, Brasil.
}

\begin{abstract}
Early detection of cervical cancer substantially increases the probability of cure. The aim of this study was to determine the prevalence and factors associated with non-participation in cervical cancer screening among women 15 to 49 years of age in Rio Grande, Southern Brazil. A representative survey was carried out using a systematic sample based on a census tract. Trained interviewers used standard, individualized, household questionnaires to interview the women. Data analysis used logistic regression according to a previously determined hierarchical framework. Among 1,302 women interviewed, 57\% had never had a Pap smear. The risk factors most closely associated with non-participation in screening were black or brown skin color, young age, low family income, low schooling, living alone, and first childbirth after 25 years of age. Coverage for early detection of cervical cancer was very low. In addition, women at high risk of developing cervical cancer were shown to have a significantly higher risk of non-participation in this type of screening.
\end{abstract}

Key words Uterine Neoplasms; Papanicolaou; Women's Health; Risk Factors; Screening

Resumo A identificação precoce do câncer de colo uterino aumenta substancialmente a sua probabilidade de cura. Este estudo mediu a prevalência e identificou alguns fatores associados à não realização de exame citopatológico de colo uterino entre mulheres em idade fértil, no Município de Rio Grande, Rio Grande do Sul, Brasil. Utilizou-se delineamento transversal, de base populacional com amostragem sistemática por conglomerados. Aplicou-se questionário individual, padronizado mediante visita domiciliar, por entrevistadores previamente treinados. Os dados foram analisados por regressão logística obedecendo a modelo hierárquico previamente determinado. Dentre as 1.302 mulheres entrevistadas, 57\% nunca se submeteram ao exame citopatológico para detecção de câncer uterino. Mulheres de cor parda ou preta, de menor idade, renda familiar e escolaridade, que estavam vivendo sem companheiros e que tiveram o primeiro parto com 25 anos ou mais de idade foram as que apresentaram as maiores razões de prevalências para a não realização deste tipo de exame. A cobertura para detecção precoce de câncer de colo uterino por meio de exame citopatológico foi muito baixa em Rio Grande. Além disso, mulheres com maior probabilidade de ter esta doença foram as que apresentaram maiores razões de prevalências à sua não realização.

Palavras-chave Neoplasias Uterinas; Papanicolaou; Saúde da Mulher; Fatores de Risco; Rastreamento 


\section{Introdução}

Cerca de $80 \%$ dos casos de câncer de colo uterino ocorrem nos países pobres (Parkin et al., 1993). Exames preventivos periódicos permitem reduzir em até $70 \%$ a sua mortalidade na população de risco (INCA, 2002; Sankaranarayanan et al., 2001). A faixa etária mais acometida vai dos 25 ao 60 anos e o seu mais importante determinante é o papilomavírus humano (Eluf Neto et al., 1994; Walboomers et al., 1999). Na América Latina, fatores reprodutivos maternos tais como início precoce de atividade sexual e comportamento sexual masculino, podem ser responsabilizados pela ocorrência de grande parte deste tipo de câncer (Eluf Neto \& Nascimento, 2001; Herrero et al., 1990). Dentre outras condições facilitadoras à sua ocorrência estão baixo nível socioeconômico, múltiplos parceiros sexuais, higiene sexual precária, ocorrência de abortos (particularmente os induzidos), alcoolismo e tabagismo. O uso de anticoncepcional oral também tem sido referido como provável fator associado à etiologia desse tipo de câncer (Drain et al., 2002; Eluf Neto \& Nascimento, 2001; Herrero et al., 1990; INCA, 2002; Robles et al., 1996).

No Brasil, o câncer de colo uterino é o segundo entre os tumores ginecológicos malignos e o terceiro mais comum na população feminina. Representa $10 \%$ de todos os cânceres malignos em mulheres (INCA, 2002). No Rio Grande do Sul, o câncer de colo uterino é a quarta maior causa de óbito entre mulheres. Na década de 90, houve, neste Estado, um aumento de $27 \%$ na ocorrência deste tipo de câncer, passando de 5,2/100 mil para 6,6/100 mil habitantes (Secretaria da Saúde do Estado do Rio Grande do Sul, 2000).

Considerando a elevada ocorrência deste tipo de câncer e, dependendo do estágio em que é detectado, do seu elevado potencial de cura a um custo aceitável pelos serviços de saúde, é licito destacar a importância de exames periódicos como estratégia para redução da mortalidade por esta causa (Drain et al., 2002; Herrero et al., 1990; INCA, 2002; Robles et al., 1996; Sankaranarayanan et al., 2001).

Este estudo buscou conhecer a prevalência de não realização de exame citopatológico de colo uterino entre mulheres de 15 a 49 anos em algum momento de suas vidas, e identificar possíveis fatores associados à sua não realização.

\section{Metodologia}

Com cerca de 200 mil habitantes, Rio Grande é o maior centro industrial do extremo Sul do Brasil. Sua economia está alicerçada na indústria petroquímica, de pescados e na atividade portuária. Três hospitais gerais, quatro ambulatórios de especialidades médicas e 26 postos de saúde constituíam o sistema público de saúde quando este estudo foi conduzido no início de 1995.

A população-alvo deste estudo foi composta por mulheres em idade fértil ( 15 a 49 anos) residentes nas zonas urbana e rural do município. O tamanho da amostra necessário para se detectar a prevalência de não realização do exame citopatológico de colo uterino entre estas mulheres no Município de Rio Grande, foi definido segundo freqüência esperada de realização de exame citopatológico de colo uterino. Estimou-se, com base em dados de Pelotas, Rio Grande do Sul, um município vizinho, que cerca de metade delas já havia realizado este exame em algum momento no passado (Dias-daCosta, 1993). Para se trabalhar com uma precisão de $5 \%$ e um erro alfa de 0,05 seria necessário entrevistar cerca de 870 mulheres. A este número foram acrescidos $30 \%$ para controle de fatores de confusão como, por exemplo, idade da mulher, renda familiar, escolaridade, paridade, etc., e $10 \%$ para possíveis perdas e recusas (Smith \& Day, 1984). Assim, o tamanho final da amostra deveria contemplar pelo menos 1.249 mulheres.

De acordo com a Fundação Instituto Brasileiro de Geografia e Estatística (IBGE, 1994), no Município de Rio Grande existiam cinco mulheres em idade fértil a cada quatro domicílios (0,8 mulher/domicílio). Portanto, visitando cerca de 2 mil domicílios seria possível encontrar em torno de 1.600 mulheres. Este número seria suficiente para obter o tamanho de amostra desejado, já excluídas aquelas com menos de 20 anos de idade que não possuem ainda indicação formal para realizar este tipo de exame. Baseado no estudo piloto e em inquéritos realizados pelo Fundo das Nações Unidas para a Infância (UNICEF) no Nordeste, estimou-se este valor em $15 \%$. Decidiu-se, a priori, que a pesquisa cobriria um terço de todos os setores censitários do município. Como Rio Grande possui 221 setores, 74 seriam incluídos no estudo. Assim, visitando 28 domicílios em cada setor totalizaria 2.072 domicílios ou cerca de 1.650 mulheres. Excluindo-se as mulheres com idade inferior a 20 anos e aquelas que relataram nunca haver mantido relação sexual, que não tinham necessidade de se submeter a tal 
exame, restariam ainda cerca de 1.400 mulheres, número suficiente para alcançar o tamanho de amostra desejado.

Esses domicílios foram escolhidos segundo amostragem sistemática por conglomerados. $\mathrm{O}$ primeiro setor foi escolhido aleatoriamente e os demais adicionando três - resultado da divisão entre o número de setores disponíveis (221) pelo desejado (74) - ao setor inicialmente sorteado. De posse de mapas dos setores fornecidos pelo escritório local do IBGE, todas as quadras foram numeradas e uma delas escolhida aleatoriamente. Em seguida, e da mesma forma, escolhia-se a esquina a partir da qual 28 domicílios foram visitados consecutivamente no sentido horário. Caso não fosse possível obter o número desejado, passava-se à quadra seguinte, dentro do mesmo setor, mais próxima ao ponto inicialmente sorteado. A definição dos setores ficou ao encargo dos coordenadores do estudo (J.A.C. e B.L.H.).

Aplicou-se questionário único, padronizado a todas as mulheres em idade fértil residentes nos domicílios sorteados. Esse questionário buscava informações sobre características demográficas, ambientais, sócio-econômicas e realização de citopatológico de colo uterino alguma vez no passado. O questionário foi aplicado por 12 estudantes dos cursos de graduação em Enfermagem e Obstetrícia e Medicina da Universidade Federal do Rio Grande, previamente treinados em técnica de entrevista durante uma semana pelos coordenadores do estudo. Esses alunos foram divididos em três equipes, cada uma delas com um supervisor. O estudo piloto foi realizado em três setores não incluídos no estudo e compreendeu a realização de 52 entrevistas completas.

O trabalho de campo foi realizado entre os meses de janeiro e abril de 1995 . Nesse período, ao final de cada dia de trabalho, cada entrevistador codificava os questionários aplicados e imediatamente os remetia a um revisor. Em seguida, esses questionários eram duplamente digitados utilizando-se o programa Epi Info 6.02. Cinco por cento das entrevistas foram parcialmente repetidas pelos coordenadores. Isto visava checar, além da sua realização, consistência entre o que havia sido anotado pelo entrevistador e o informado pela entrevistada, não sendo observada nenhuma diferença importante.

Foram estudadas as seguintes variáveis: cor da pele (definida pelo próprio entrevistador em branca, parda/morena e preta), idade (em anos completos no momento da entrevista), viver com companheiro no mesmo domicílio (independente de a união ser formal ou não), renda familiar (valor recebido por todos os morado- res do domicílio no mês imediatamente anterior à entrevista), escolaridade (em anos completos e com aprovação), idade de início das relações sexuais (idade em anos completos quando manteve relação sexual pela primeira vez), uso de anticoncepcional oral no mês anterior à entrevista, idade por ocasião do primeiro parto (idade em anos completos quando teve o primeiro filho, independente de ter nascido vivo ou morto), número de filhos nascidos vivos, e ocorrência de aborto espontâneo ou provocado em algum momento no passado.

Considerou-se como variável dependente à não realização de citopatológico de colo uterino em algum momento no passado. Logo, exames realizados em decorrência de alguma doença, sinal ou sintoma não foram considerados neste estudo. Também, mulheres com idade inferior a 20 anos que nunca mantiveram relação sexual foram excluídas desta análise. Isto se deve ao fato de, entre estas, não haver indicação formal para realização deste exame.

A análise inicial teve por objetivo medir o grau de associação entre possíveis fatores de risco (idade, renda familiar, escolaridade, uso de métodos contraceptivos, etc.) e o desfecho (não realização de citopatológico de colo uterino). Estas associações foram medidas usando-se o cálculo da razão de prevalências (e de seu intervalo de confiança de 95\%) e do teste do qui-quadrado para tabelas de contingência (Szklo \& Nieto, 2000). A análise multivariada, realizada por meio de regressão logística não condicional, obedeceu ao modelo previamente estabelecido e mostrado na Tabela 1. De acordo com esse modelo, algumas variáveis seriam sobredeterminantes em relação às demais (Victora et al., 1997). Por exemplo, viver com companheiro, situado no primeiro nível, poderia influenciar todas as outras variáveis. O segundo nível foi constituído por renda familiar e escolaridade da mulher, no terceiro, idade de início das relações sexuais e no quarto nível, uso de anticoncepcional oral. O nível seguinte incluiu idade por ocasião do primeiro parto, número de filhos nascidos vivos e ocorrência prévia de aborto. A significância estatística da inclusão de cada variável no modelo foi aferida mediante regressão de Poisson com estimativa robusta das variáveis. O modelo final incluiu todas as variáveis que, ao entrarem na equação - isto é, já ajustadas para variáveis dos níveis superiores e do mesmo nível - apresentam um valor de p igual ou inferior a 0,10 . Cada variável ordinal - por exemplo, escolaridade da mulher em grupos - foi também testada para tendência linear que, em caso de haver significância mas sem desvio da linearidade, a variável era incluída no 
Modelo hierárquico para não realização de citopatológico de colo uterino alguma vez, entre mulheres em idade fértil. Rio Grande, Rio Grande do Sul, Brasil, 1995.

\begin{tabular}{ll}
\hline Nível * & Variáveis \\
\hline Primeiro & Vive com companheiro. \\
Segundo & Renda familiar e escolaridade da mulher. \\
Terceiro & Idade de início das relações sexuais. \\
Quarto & Uso de contraceptivo oral. \\
Quinto & Idade no primeiro parto, número de filhos nascidos vivos e ocorrência \\
& de aborto em algum momento no passado. \\
Desfecho & Não realização de exame citopatológico para câncer de colo uterino.
\end{tabular}

* Todas as variáveis presentes neste modelo foram ajustadas para "idade da mulher"

e "cor da pele" porque, embora não sejam sobredeterminantes em relação às demais,

mostraram-se significativamente associadas ao desfecho.

modelo como um componente linear (Szklo \& Nieto, 2000). Valores desconhecidos foram desconsiderados nesta análise. A variável mais afetada por este problema foi renda familiar onde $32(4,5 \%)$ não souberam informá-la. Entre as demais variáveis, este valor foi menor que $1 \%$.

\section{Resultados}

Nos 2.072 domicílios visitados residiam 1.471 mulheres em idade fértil. Doze delas não foram encontradas e três recusaram-se a responder ao questionário mesmo após uma terceira tentativa por entrevistadores diferentes, incluindo-se um dos coordenadores deste estudo (J.A.C.). Isto representa $1,2 \%$ de perdas. Cento e cinqüenta e quatro delas possuíam idade inferior a 20 anos e, conforme relataram, nunca haviam mantido relação sexual e, por esta razão, foram excluídas desta análise. Assim, o denominador deste estudo foi constituído por 1.302 mulheres com idade entre 15 e 49 anos.

Cerca de $10 \%$ dessas mulheres possuíam entre 15 e 19 anos, $24 \%$ entre 40 e 49; $84 \%$ eram de cor branca e $72 \%$ viviam com companheiro. Quarenta e dois por cento exerceram trabalho remunerado nos últimos 12 meses anteriores à entrevista, $27 \%$ possuíam renda familiar inferior a dois salários mínimos mensais (SMM) e, para metade delas, a renda familiar era igual ou superior a três SMM. Cinco por cento das entrevistadas não sabiam ler nem escrever, apenas assinavam o nome, e cerca de um terço possuía nove anos ou mais de escolaridade completa com aprovação.

Noventa e sete por cento delas já haviam mantido relação sexual sendo que, para $70 \%$, isto ocorreu antes dos 20 anos de idade. Dentre as que já tinham iniciado vida sexual, $68 \%$ haviam utilizado algum método contraceptivo no mês anterior à entrevista. O método mais comumente referido foi anticoncepcional oral (69\%), seguido por ligadura de trompas (19\%). Dentre as que não utilizaram nenhum método, os motivos mais citados foram não ter vida sexual ativa (64\%) e querer engravidar (20\%). Vinte e um por cento de todas as entrevistadas já tiveram pelo menos um aborto, um terço dos quais sendo provocado. O misoprostol (Citotec) e o uso de sondas foram os métodos de indução mais freqüentemente utilizados com $27 \%$ e $20 \%$, respectivamente.

Dentre as 1.302 mulheres incluídas neste estudo, $57 \%$ nunca realizaram exame para detecção precoce de câncer de colo uterino. A análise bruta mostra que estiveram associadas à não realização deste exame as seguintes variáveis: cor da pele, idade, viver com companheiro, renda familiar, escolaridade, idade de início das relações sexuais, uso de anticoncepcional oral no mês anterior à entrevista, idade por ocasião do primeiro parto, número de filhos nascidos vivos, e ocorrência de aborto prévio (Tabelas 2 e 3).

Ao se ajustar esta análise conforme modelo hierárquico proposto na Tabela 1 , perderam a significância estatística $(\mathrm{p}>0,05)$ as seguintes variáveis: idade de início das relações sexuais, uso de método contraceptivo no mês anterior à entrevista, número de filhos nascidos vivos $\mathrm{e}$ ocorrência de aborto em algum momento no passado. Mulheres de cor parda ou preta apresentaram razão de prevalências (RP) de 1,3 (1,21,4) maior à não prevenção de câncer de colo uterino em relação àquelas de cor branca. Quanto menor a idade, maior a probabilidade de não 
Modelo de regressão logística hierárquica múltipla para efeito de algumas variáveis sobre a não realização de exame para detecção precoce de câncer de colo uterino alguma vez, entre mulheres em idade fértil. Rio Grande, Rio Grande do Sul, Brasil, 1995.

\begin{tabular}{|c|c|c|c|}
\hline \multirow[t]{2}{*}{ Variável } & \multirow{2}{*}{$\begin{array}{l}\text { Número de mulheres } \\
\text { por categoria e }(\%) \text { de não } \\
\text { examinadas }(n=1.302)\end{array}$} & \multicolumn{2}{|c|}{$\begin{array}{l}\text { Razão de prevalências } \\
\text { com IC95\% }\end{array}$} \\
\hline & & Bruta & Ajustada \\
\hline Cor da pele & & $P<0,001$ & $P<0,001 a$ \\
\hline Parda ou preta & $146(70,9)$ & $1,29(1,17-1,44)$ & $1,30(1,18-1,44)$ \\
\hline Branca & $599(54,7)$ & 1,00 & 1,00 \\
\hline Idade da mulher (anos) & & $P<0,001$ & $P<0,001^{*} b$ \\
\hline $15-19$ & $86(86,7)$ & $1,82(1,59-2,09)$ & $1,83(1,60-2,09)$ \\
\hline $20-29$ & $255(67,3)$ & $1,40(1,23-1,60)$ & $1,40(1,23-1,59)$ \\
\hline $30-39$ & $235(50,0)$ & $1,04(0,91-1,21)$ & $1,04(0,90-1,19)$ \\
\hline $40-49$ & $169(47,7)$ & 1,00 & 1,00 \\
\hline Vive com companheiro & & $P<0,001$ & $P<0,05 c$ \\
\hline Sim, atualmente & $510(54,2)$ & $0,75(0,68-0,82)$ & $0,86(0,77-0,95)$ \\
\hline Sim, no passado & $60(50,0)$ & $0,69(0,57-0,84)$ & $0,83(0,68-1,02)$ \\
\hline Solteira & $175(72,6)$ & 1,00 & 1,00 \\
\hline Renda familiar mensal (salários mínimos) & & $P<0,001$ & $P<0,05^{\star} d$ \\
\hline$<1$ & $97(68,3)$ & $1,62(1,37-1,92)$ & $1,27(1,05-1,52)$ \\
\hline $1-1,9$ & $147(68,1)$ & $1,61(1,38-1,89)$ & $1,29(1,09-1,52)$ \\
\hline $2-2,9$ & $159(69,7)$ & $1,65(1,42-1,93)$ & $1,39(1,18-1,63)$ \\
\hline $3-5,9$ & $164(50,8)$ & $1,20(1,02-1,42)$ & $1,07(0,91-1,27)$ \\
\hline 6 ou + & $142(42,1)$ & 1,00 & 1,00 \\
\hline Escolaridade (em anos completos) & & $P<0,001$ & $P<0,05^{\star}$ e \\
\hline Nenhum & $50(73,5)$ & $1,66(1,38-1,98)$ & $1,46(1,20-1,78)$ \\
\hline $1-4$ & $208(63,2)$ & $1,42(1,24-1,63)$ & $1,30(1,12-1,51)$ \\
\hline $5-8$ & $309(61,3)$ & $1,38(1,21-1,57)$ & $1,23(1,07-1,42)$ \\
\hline 9 ou + & $178(44,4)$ & 1,00 & 1,00 \\
\hline
\end{tabular}

* Teste para tendência linear de proporções (uni-caudal).

Modelos da análise ajustada: (a) idade da mulher; (b) cor da pele; (c) idade da mulher +

cor da pele; (d) idade da mulher + cor da pele + vive com companheiro + escolaridade;

(e) idade da mulher + cor da pele + vive com companheiro + renda familiar.

realização deste tipo de exame. Esta RP foi de $1,8(1,6-2,1)$ maior entre adolescentes (menos de 20 anos), quando comparadas a mulheres com 40 anos ou mais. Mulheres que estavam vivendo com companheiro no momento da entrevista, mostraram maior probabilidade de realização de citopatológico de colo uterino em relação às que viviam sozinhas. Mulheres de menor renda apresentaram razão de prevalências cerca de $30 \%$ maior à não prevenção deste tipo de câncer em relação àquelas com renda igual ou superior a 6 SMM. A RP entre mulheres não alfabetizadas foi 1,5 (1,2-1,8) quando comparadas àquelas com nove anos ou mais de escolaridade completos. Em relação a este mesmo grupo de mulheres, a razão de prevalências para mulheres com escolaridade entre 1 e 4 anos foi de 1,3 (1,1-1,5). A razão de prevalências para não realização deste exame entre mulheres que tiveram o primeiro parto antes dos 25 anos de idade foi cerca de $20 \%$ menor em relação às demais. As Tabelas 2 e 3 mostram estes dados.

\section{Discussão}

Este estudo mostra baixa realização de exame citopatológico de colo uterino entre as riograndinas em idade fértil. Mulheres de cor parda ou preta, de menor idade, renda familiar e escolaridade, que não estavam vivendo com companheiro no momento da entrevista e que tiveram 
Modelo de regressão logística hierárquica múltipla para efeito de algumas variáveis sobre a não realização de exame para detecção precoce de câncer de colo uterino alguma vez, entre mulheres em idade fértil. Rio Grande, Rio Grande do Sul, Brasil, 1995.

\begin{tabular}{|c|c|c|c|}
\hline \multirow[t]{2}{*}{ Variável } & \multirow{2}{*}{$\begin{array}{l}\text { Número de mulheres } \\
\text { por categoria e }(\%) \text { de não } \\
\text { examinadas }(n=1.302 \text { ) }\end{array}$} & \multicolumn{2}{|c|}{$\begin{array}{l}\text { Razão de prevalências } \\
\text { com IC } 95 \%\end{array}$} \\
\hline & & Bruta & Ajustada \\
\hline Idade (em anos) de início das relações & $\mathrm{n}=1.267$ & $P<0,001$ & $P=0,16^{*} f$ \\
\hline Até 15 & $140(70)$ & $1,11(0,97-1,28)$ & $1,00(0,86-1,18)$ \\
\hline $16-17$ & $185(59,8)$ & $1,22(1,07-1,40)$ & $1,02(0,88-1,17)$ \\
\hline $18-19$ & $194(54,5)$ & $1,43(1,25-1,64)$ & $1,00(0,87-1,16)$ \\
\hline 20 ou + & $196(48,9)$ & 1,00 & 1,00 \\
\hline $\begin{array}{l}\text { Uso de anticoncepcional } \\
\text { no mês anterior à entrevista }\end{array}$ & $\mathrm{n}=1.282$ & $P<0,001$ & $P=0,19 f$ \\
\hline Não & $173(67,1)$ & $1,24(1,12-1,38)$ & $1,11(0,96-1,28)$ \\
\hline Outro & $70(54,3)$ & $1,00(0,85-1,19)$ & $0,93(0,75-1,14)$ \\
\hline Sim & $484(54,1)$ & 1,00 & 1,00 \\
\hline $\begin{array}{l}\text { Idade (em anos) por ocasião } \\
\text { do primeiro parto }\end{array}$ & $n=939$ & $P<0,001$ & $P<0,05^{\star} g$ \\
\hline$<20$ & $125(48,6)$ & $0,68(0,58-0,79)$ & $0,81(0,67-0,99)$ \\
\hline $20-24$ & $217(51,2)$ & $0,71(0,63-0,80)$ & $0,78(0,66-0.94)$ \\
\hline 25 ou + & $185(71,7)$ & 1,00 & 1,00 \\
\hline Filhos nascidos vivos & $\mathrm{n}=1.302$ & $P<0,01$ & $P=0,23 h$ \\
\hline Nenhum & $361(62,5)$ & $0,70(0,61-0,80)$ & $0,85(0,68-1,05)$ \\
\hline 1 & $187(51,9)$ & $0,58(0,49-0,68)$ & $0,83(0,62-1,10)$ \\
\hline 2 & $171(51,0)$ & $0,57(0,48-0,67)$ & $0,83(0,63-1,10)$ \\
\hline 3 ou + & $26(89,7)$ & 1,00 & 1,00 \\
\hline Ocorrência de aborto prévio & $\mathrm{n}=1.302$ & $P<0,001$ & $P=0,14 i$ \\
\hline Sim & $610(59,3)$ & $1,20(1,06-1,37)$ & $1,09(0,92-1,26)$ \\
\hline Não & $135(49,3)$ & 1,00 & 1,00 \\
\hline
\end{tabular}

* Teste para tendência linear de proporções (uni-caudal).

Modelos da análise ajustada: $(f)$ idade da mulher + cor da pele + vive com companheiro + renda familiar + escolaridade; $(\mathrm{g})$ idade da mulher + cor da pele + vive com companheiro + renda familiar + escolaridade + filhos nascidos vivos + ocorrência de aborto prévio; (h) idade da mulher + cor da pele + vive com companheiro + renda familiar + escolaridade + idade no primeiro parto + ocorrência de aborto prévio; (i) idade + cor da pele + vive com companheiro

+ renda familiar + escolaridade + filhos nascidos vivos + idade no primeiro parto.

o primeiro parto com 25 anos ou mais de idade, apresentaram razões de prevalências significativamente maiores à não realização de exame citopatológico para detecção de câncer de colo uterino em relação às demais mulheres.

Ao se interpretar estes resultados, é preciso considerar pelo menos três aspectos: (1) estudos que tratam desse assunto partem do pressuposto que esses exames são amplamente conhecidos pelas mulheres; (2) desconsideram o fato de elas superestimarem a freqüência com que os realizam e de subestimarem o tempo decorrido em relação ao exame mais recente; e (3) não levam em consideração a pressão social que elas sofrem pela adoção de comportamento correto em relação a certas práticas em saúde, particularmente as preventivas (Nascimento et. al., 1996). Não há como contornar estas limitações utilizando-se de um delineamento transversal. Também vale lembrar que, até o presente momento nenhum estudo publicado sobre o assunto contornou satisfatoriamente estes problemas e nem por isso deixou-se de planejar ações em saúde e implementar programas nesta área.

Ainda neste estudo, a variável cor da pele, avaliada pelo próprio entrevistador, que se mostrou fortemente associada à não realização de citopatológico de colo uterino, pode apresentar algum grau de subjetividade. A fim de que isto 
não afetasse de forma importante os nossos resultados, os entrevistadores foram orientados para classificar por exclusão, quem não era de cor branca ou preta, que são mais fáceis de definir, foram classificadas como pardas. Procedemos desta forma porque esta subjetividade seria maior se o próprio entrevistado classificasse a sua cor. Além disso, esse tipo de classificação é adotado por quase todos os estudos epidemiológicos.

O Instituto Nacional de Câncer (INCA) recomenda o exame citopatológico para câncer de colo uterino a toda mulher com idade entre 20 e 60 anos e que possua vida sexual ativa. Inicialmente, o exame deve ser feito a cada três anos. Em caso de dois resultados negativos para displasia ou neoplasia, um novo exame deverá ser repetido uma vez a cada três anos (INCA, 2002). Cinqüenta e sete por cento das riograndinas em idade fértil nunca realizaram um único exame citopatológico de colo uterino em qualquer momento da vida. Ainda que a faixa etária não seja exatamente a mesma, este valor é muito superior aos 10\% referidos pelo Ministério da Saúde (MS) para o Brasil em 1998, mas inferior aos $69 \%$ observados para o Município de São Paulo em 1987, oito anos antes deste estudo (Nascimento et al., 1996) e os 65\% verificados na mesma época para o Município de Pelotas (Dias-da-Costa et al., 1998).

A cobertura para detecção de câncer de colo uterino em Rio Grande é muito baixa. Preocupa ainda o fato de, no geral, as mulheres com maiores riscos de adquirir esta doença terem apresentado as maiores razões de prevalência à não realização deste tipo de exame. Isto é particularmente grave porque são exatamente essas mulheres que mais se beneficiariam deste procedimento. Mesmo o estudo de Pelotas, que mostrou uma freqüência muito maior de realização de citopatológico de colo uterino, revelou que a realização deste tipo de exame foi cerca de $20 \%$ menor entre mulheres com maior risco para câncer de colo uterino (Dias-da-Costa et al., 1998).

Estudo qualitativo realizado na Cidade do México e em Oaxaca, sul do país, revelou como limitantes à realização de citopatológico de colo uterino as seguintes barreiras: (1) falta de conhecimento quanto aos fatores facilitadores (ou causadores) do câncer de colo uterino; (2) desconhecimento quanto a existência deste tipo de exame e/ou de sua utilidade; (3) concepção de que câncer é uma doença fatal e que, portanto, não adianta preveni-lo; (4) dificuldade na relação medição-paciente; (5) não é visto como um exame prioritário dentro das tantas necessidades em saúde que apresentam; (6) oposição do companheiro/marido à realização deste tipo de exame; (7) rejeição (ou tabu) por parte da mulher por se tratar de um exame pélvico; (8) longo tempo de espera até a obtenção do resultado do exame; e (9) idéia de que se trata de um exame de custo elevado (Lazcano-Ponce et al., 1999). Embora as populações destes dois estudos apresentem alguma diferença quanto a características demográficas e ao tipo de abordagem utilizada, o estudo aqui apresentado é quantitativo. Também, é lícito supor que muitas destas barreiras apontadas acima podem ser aplicáveis às riograndinas em idade fértil. Isto porque a rede de serviços de saúde no município é abundante - metade dessas mulheres reside a menos de $1 \mathrm{~km}$ do serviço de saúde mais próximo de suas casas (Cesar \& Horta, 1997) e este tipo de cuidado em saúde, segundo a coordenação local do programa, é oferecido em todos os postos de saúde da rede pública municipal.

Os resultados aqui apresentados revelam que é preciso aumentar a cobertura de citopatológico de colo uterino no Município de Rio Grande. No entanto, é preciso envidar esforços no sentido de alcançar essas mulheres, principalmente aquelas com maior risco de adoecer e morrer por estas causa. Para isso, campanhas poderiam ser realizadas utilizando-se os meios de comunicação, que também são fartos na localidade, anúncios nos próprios serviços de saúde e, sobretudo, com a implementação do Programa Saúde da Família (PSF), por meio de reuniões na comunidade pelas equipes de saúde. A busca ativa de mulheres pelo agente comunitário de saúde durante as visitas regulares que ele realiza, pode constituir-se em um mecanismo bastante efetivo e eficiente. Também deveriam aproveitar melhor as oportunidades oferecidas. Por exemplo, em Rio Grande, 90\% das mulheres em idade fértil fazem pelo menos um consulta de pré-natal (Cesar \& Horta, 1997). Essas consultas poderiam ser usadas também para a realização desses exames que fazem parte dos cuidados pré-natais. Somente nesta situação seria possível diagnosticar cerca de metade dos casos em um estágio onde o percentual de cura é extremamente alto (Robles et al., 1996). Finalmente, especial atenção deveria ser dada às mulheres de maior idade, visto que essas diminuem sua freqüência de visitas ao serviço à medida que se afastam do período fértil, exatamente quando a incidência desta doença aumenta (Robles et al., 1996).

Em se tratando de detectar precocemente câncer de colo uterino em Rio Grande, o serviço local de saúde mostrou-se pouco efetivo e desigual. Pouco efetivo porque cobriu menos 
da metade das mulheres em idade fértil naquele município, e desigual porque o acesso a esses exames variou conforme algumas características das usuárias. É preciso estender este benefício a todas as mulheres, mas principalmente àquelas de cor parda ou preta, de menor idade, renda familiar e escolaridade, que não estão vivendo com companheiro e que têm o primeiro parto com 25 anos ou mais de idade. Esta medida poderia contribuir para a redução da mortalidade por uma doença relativamente freqüente e quase sempre evitável.

\section{Referências}

CESAR, J. A. \& HORTA, B. L., 1997. Desigualdade e Perversidade: Epidemiologia do Adoecer no Extremo Sul do Brasil. Rio Grande: Editora da FURG.

DIAS-DA-COSTA, J. S., 1993. Utilização de Serviços de Saúde em Pelotas, RS. Dissertação de Mestrado, Pelotas: Faculdade de Medicina, Universidade Federal de Pelotas.

DIAS-DA-COSTA, J. S.; D'ELIA, P. B.; MANZOLLI, P. \& MOREIRA, M. R., 1998. Cobertura para exame citopatológico de colo uterino em Pelotas, RS. Revista Panamericana de Salud Pública, 3:308-313.

DRAIN, P. K.; HOLMES, K. K.; HUGHES, J. P. \& KOUTSKY, L. A., 2002. Determinants of cervical cancer rates in developing countries. International Journal of Cancer, 100:199-205.

ELUF NETO, J.; BOOTH, M.; MUNOZ, N.; BOSCH, F. X.; MEIJER, C. J. \& WALBOOMERS, J. M., 1994. Human papillomavirus and invasive cervical cancer in Brazil. British Journal of Cancer, 69:114-119.

ELUF NETO, J. \& NASCIMENTO, C. M., 2001. Cervical cancer in Latin America. Seminars in Oncology, 28:188-197.

HERRERO, R.; BRINTON, L. A.; REEVES, W. C.; BRENES, M. M.; TENORIO, F.; DE BRITTON, R. C.; GAITAN, E.; MONTALVAN, P.; GARCIA, M. \& RAWLS, W. E., 1990. The risk factors of invasive carcinoma of the cervix uteri in Latin America. Boletín de la Oficina Sanitaria Panamericana, 109:6-26.

IBGE (Fundação Instituto Brasileiro de Geografia e Estatística), 1994. Indicadores Sociais: Censo Demográfico, Rio Grande do Sul 1991, 1994. Rio de Janeiro: IBGE.

INCA (Instituto Nacional de Câncer), 2002. Câncer de Colo de Útero. 5 Fevereiro $2002<$ http:/ / www.inca. org.br/cancer/tipos/utero.html $>$.

LAZCANO-PONCE, E. C.; CASTRO, R.; ALLEN, B.; NAJERA, P.; ALONSO-DE-RUIZ, P. A. \& HERNANDEZAVILA, M., 1999. Barriers to eraly detection of cervical-uterine cancer in Mexico. Journal of Women's Health, 8:399-408.

\section{Agradecimentos}

À Secretaria de Saúde e Bem-Estar da Prefeitura Municipal de Rio Grande, Rio Grande do Sul, e à Fundação de Amparo à Pesquisa do Rio Grande do Sul (FAPERGS) pelo apoio financeiro e logístico à realização deste estudo; às mulheres que participaram desta análise e ao Dr. S. Vohra, do Health Service Research Unit, London School of Hygiene \& Tropical Medicine, pelos comentários.
NASCIMENTO, C. M. R.; ELUF NETO, J. \& REGO, R. A., 1996. Pap test coverage in São Paulo municipality and characteristics of the women tested. Bulletin of Pan American Health Organization, 30:302-311.

PARKIN, D.; PISANI, P. \& FERLAY, J., 1993. Estimates of the worldwide incidence of eighteen major cancers in 1985. International Journal of Cancer, 54: 594-606.

ROBLES, S. C.; WHITE, F. \& PERUGA, A., 1996. Trends in cervical cancer mortality in the Americas. Bulletin of Pan American Health Organization, 30: 291-301.

SANKARANARAYANAN, R.; BUDUKH, A. M. \& RAJKUMAR, R., 2001. Effective programmes for cervical cancer in low and middle income developing countries. Bulletin of the World Health Organization, 79:954-962.

SECRETARIA DA SAÚDE DO ESTADO DO RIO GRANDE DO SUL, 2001. Estatísticas de Saúde-Mortalidade 2000, 2001. v. 25. Porto Alegre: Secretaria da Saúde do Estado do Rio Grande do Sul.

SMITH, P. G. \& DAY, N. E., 1984. The design of casecontrol studies: The influence of confounding and interactions effects. International Journal of Epidemiology, 13:356-365.

SZKLO, M. \& NIETO, J. F., 2000. Epidemiology Beyond the Basics. Gaithersburg: Aspen Publications.

VICTORA, C. G.; HUTLLY, S. R.; FUCHS, S. C. \& OLINTO, M. T. A., 1997. The role of conceptual frameworks in epidemiological analysis: A hierarchical approach. International Journal of Epidemiology, 26:224-227.

WALBOOMERS, J. M.; JACOBS, M. V.; MANOS, M. M.; BOSCH, F. X.; KUMMER, J. A.; SHAH, K. V; SNIJDERS, P. J.; PETO, J.; MEIJER, C. J. \& MUÑOZ, N., 1999. Human papilomavirus is a necessary cause of invasive cervical cancer worldwide. Journal of Pathology, 189:12-19.

Recebido em 20 de fevereiro de 2002

Versão final reapresentada em 27 de março de 2003

Aprovado em 19 de maio de 2003 